laterolateral dimensions at level3, hyoid bone position, ESS and neck circumference.

Conclusion In OSA , significant alteration of anthropometry of upper airway occurs and is associated with severity of OSA.

\section{P218 THE EFFECTS OF SUPPLEMENTAL OXYGEN ON BLOOD PRESSURE IN OBSTRUCTIVE SLEEP APNOEA DURING CPAP WITHDRAWAL}

${ }^{1} \mathrm{CD}$ Turnbull, ${ }^{2} \mathrm{~N}$ Petousi, ${ }^{1} \mathrm{D}$ Sen, ${ }^{1} \mathrm{JR}$ Stradling, ${ }^{3} \mathrm{M}$ Kohler. ${ }^{1} \mathrm{NIHR}$ Biomedical Research Centre, University of Oxford, Oxford, UK; ${ }^{2}$ Nuffield Department of Medicine, University of Oxford, Oxford, UK; ${ }^{3}$ Division of Pulmonology and Sleep Disorders Centre, University Hospital of Zurich, Zurich, Switzerland

10.1136/thoraxjnl-2017-210983.360

Introduction Intermittent hypoxia and/or intermittent arousals are thought to be the two key pathological mechanisms in the development of hypertension in obstructive sleep apnoea (OSA). We aimed to investigate the effect of abolishing the hypoxia on the rise in blood pressure (BP) that has been shown to follow continuous positive airway pressure (CPAP) withdrawal in patients with OSA. In addition, we explored the effect of supplemental oxygen on obstructive events during sleep.

Abstract P218 Table 1 The results of early morning blood pressure and overnight sleep studies on supplemental oxygen and supplemental air. Paired t-tests were used for home early morning blood pressure and heart rate measurements. Wilcoxon rank tests were used for home respiratory sleep study derivatives which were not normally distributed

Supplemental air Supplemental

oxygen

Home early morning blood pressure and heart rate measurements

Baseline Follow- Baseline Follow- Mean difference in

up change in BP $(95 \%$ confidence interval)

$\begin{array}{lllllll}\text { Mean } & 129.2 & 136.1 & 129.6 & 129.8 & -6.6(-11.3 \text { to }-1.9) & \mathbf{0 . 0 0 8}\end{array}$

systolic BP $\quad \pm 14.1 \quad \pm 14.9 \quad \pm 15.1 \quad \pm 13.6$

$(\mathrm{mmHg})$

$\begin{array}{lllllll}\text { Mean } & 78.3 & 85.3 & 79.3 & 81.6 & -4.6(-7.8 \text { to }-1.5) & 0.006\end{array}$

diastolic BP $\quad \pm 7.8 \quad \pm 9.6 \quad \pm 8.0 \quad \pm 8.0$

(mmHg)

$\begin{array}{lllllll}\text { Mean heart } & 61.7 & 64.9 & 61.9 & 64.1 & -1.0(-3.9 \text { to }+1.9) & 0.50\end{array}$

rate $(\mathrm{bpm}) \quad \pm 8.3 \quad \pm 8.9 \quad \pm 9.4 \quad \pm 9.1$

Home respiratory sleep study derivatives

Supplemental air Supplemental Difference in medians $p$

oxygen

AHI (/hour) $34.4(22.7-44.4) \quad 30.4(23.6-42.6) \quad-3.6 \quad 0.98$

ODI (/hour) $31.4(21.0-49.2) \quad 4.5(1.1-19.0) \quad-28.0 \quad 0.001$

$\begin{array}{lllll}\text { Mean } & 93.7(92.1-95.2) & 98.0(96.6-98.6) & +4.4 & 0.001\end{array}$

oxygen

saturations

(\%)

length

(mins)

Methods Patients with OSA, established on CPAP $\geq 1$ year, and with $\geq 4$ hours/ night usage, underwent a week of screening oximetry and were eligible if they had a nocturnal oxygen desaturation index $\geq 4 \%$ (ODI) of $<10$ on 3 nights on CPAP, and an ODI $\geq 20$ on at least 1 of 4 nights off CPAP. Patients then received overnight supplemental oxygen or air (via real or sham concentrators) at a flow rate of $5 \mathrm{l} / \mathrm{min}$ during 2 weeks off CPAP. After at least two weeks 'washout' back on CPAP, subjects crossed over. Treatment order was randomised. The primary outcome was early morning home BP which was recorded daily in triplicate, and averaged over the penultimate three mornings prior to each patient visit. Patients underwent overnight home respiratory sleep studies (including nasal airflow) on night 14 of each treatment arm. Differences between treatment arms were analysed using paired t-tests or Wilcoxon rank tests as appropriate.

Results Twenty-five patients completed the study. Their mean \pm standard deviation age was $63 \pm 7$ years, mean BMI was $35.3 \pm 6.7 \mathrm{~kg} / \mathrm{m}^{2}$, median (interquartile range) ODI at diagnosis was 48/hour $(25,68)$, and 21 (84\%) were male. Table 1 shows the Results of the primary outcome and the overnight sleep studies.

Discussion Supplemental oxygen abolished the rise in early morning blood pressure during CPAP withdrawal when compared to supplemental air. As expected, supplemental oxygen substantially attenuated intermittent hypoxia and had only a small non-significant effect on the apnoea hypopnoea index. Thus intermittent hypoxia appears to be the dominant determinant of the rise in morning blood pressure seen in patients with OSA, rather than any other consequence of the obstructive events.

\section{P219 THE USE OF ORAL MODAFINIL IN CHRONIC OBSTRUCTIVE PULMONARY DISEASE PATIENTS WITH CHRONIC HYPERCAPNIC RESPIRATORY FAILURE}

VA Varney, G Quirke, C Kearon, S Adeyemo, H Parnell. Respiratory Department, St Helier Hospital, Surrey, UK

\subsection{6/thoraxjnl-2017-210983.36}

Hypercapnic respiratory failure (HRF) is common in advanced COPD. The standard treatment is usually nasal ventilation (NIV) to reduce the blood carbon dioxide level acutely or regular nocturnal use in chronic cases. Not all patients tolerate such treatment and failures occur. Published studies are conflicting showing variable benefit and a 2013 Cochrane review concluded that there was no evidence of significant benefit in any of the measured parameters. ${ }^{1}$ Other studies suggest a reduction in re-admission and death at 1 year between regular nocturnal NIV use and standard care.

We have used oral modafinil $200 \mathrm{mg} /$ day as a respiratory stimulant for chronic HRF in COPD without NIV. We present the data from the first 11 cases (6 out of study and 7 in our current open randomised crossover study). The study patients had documented HRF for 6-12 months with $\mathrm{PaCO}_{2} \geq 6.5$ before entry but refused NIV. We present the data at baseline and day 10 and 40 of modafinil, including mean arterial oxygen and carbon dioxide, Daytime and overnight oxygen saturations along with spirometry. We compare these Results with 2 published studies of NIV reported, one after 4 weeks $^{2}$ and the other 12 months. $^{3}$

The study showed a mean improvement by day 40 of $+1.8 \mathrm{kPa}$ in $\mathrm{PaO}_{2}$ and a reduction in $\mathrm{PaCO}_{2}$ by $2.7 \mathrm{kPa}$. Daytime saturations, improved by $+15 \%$ and overnight 